\title{
Estresse entre graduandos de enfermagem de uma universidade pública
}

\author{
Nayanne Ingrid Farias Mota ${ }^{1}$ \\ Estela Rodrigues Paiva Alves ${ }^{2}$ \\ Gerlaine de Oliveira Leite ${ }^{3}$ \\ Brena Stefani Meira Acioly de Sousa ${ }^{4}$ \\ Maria de Oliveira Ferreira Filha ${ }^{5}$ \\ Maria Djair Dias ${ }^{5}$
}

\begin{abstract}
Objetivo: Estimar o nível de estresse e a sintomatologia apresentada em acadêmicos de enfermagem de uma universidade pública. Método: Estudo transversal e quantitativo envolvendo 151 graduandos de enfermagem selecionados a partir de um sorteio baseado na lista de alunos matriculados. Foi utilizado o inventário de sintomas do stress de Lipp versão para adultos. Os dados foram analisados e apresentados descritivamente por meio de distribuições absolutas e percentuais, utilizando software estatístico R. Resultados: 49,7\% dos alunos apresentaram sintomas de estresse, com a maioria de indivíduos cursando o $8^{\circ}$ e $9^{\circ}$ períodos. Os sintomas psicológicos foram mais presentes (50,7\%). Conclusão: Recomendase implementação de ferramentas de tecnologia leve do cuidado que apresentam baixo custo e alto poder resolutivo, para melhor combate ao estresse.
\end{abstract}

Descritores: Enfermagem; Estudantes de Enfermagem; Estresse Psicológico; Saúde Mental.

\footnotetext{
${ }^{1}$ Enfermeira.

${ }^{2}$ Doutoranda, Universidade Federal da Paraíba, João Pessoa, PB, Brasil.

${ }^{3}$ Mestranda, Universidade Federal de Pernambuco, Recife, PE, Brasil.

${ }^{4}$ Mestranda, Universidade Federal da Paraíba, João Pessoa, PB, Brasil.

${ }^{5} \mathrm{PhD}$, Professor Associado, Universidade Federal da Paraíba, João Pessoa, PB, Brasil
} 


\section{Stress among nursing students at a public university}

Objective: To estimate the level of stress and symptomatology reported in nursing students from a public university. Method: This is a transversal and quantitative study involving 151 nursing students selected from a draw based on the list of students enrolled. The adult version of the Lipp's inventory of symptoms of stress was used. Data were analyzed and descriptively presented by absolute and percentage distributions using the statistical software R. Results: $49.7 \%$ of students showed symptoms of stress, and most students were attending the 8 th and 9th periods. Psychological symptoms were more present (50.7\%). Conclusion: Implementing lightweight care technology tools is recommended, at low cost and high resolute power, to better combat stress.

Descriptors: Nursing; Students Nursing; Stress Psychological; Mental Health.

\section{Estrés entre graduandos de enfermería de una universidad pública}

Objetivo: Estimar el nivel de estrés y la sintomatología presentada en académicos de enfermería de una universidad pública. Método: Estudio transversal y cuantitativo envolviendo 151 graduandos de enfermería seleccionados desde un sorteo basado en la lista de alumnos matriculados. Fue utilizado el inventario de síntomas del estrés de Lipp versión para adultos. Los datos fueron analizados y presentados descriptivamente por medio de distribuciones absolutas y porcentuales, utilizando software estadístico R. Resultados: $49,7 \%$ de los alumnos presentaron síntomas de estrés, con la mayoría de individuos cursando el $8^{\circ}$ y $9^{\circ}$ períodos. Los síntomas psicológicos fueron más presentes (50,7\%). Conclusión: Se recomienda implementación de herramientas de tecnología lleve del cuidado que presentan bajo coste y alto poder resolutivo, para mejor combate al estrés.

Descriptores: Enfermería; Estudiantes de Enfermería; Estrés Psicológico; Salud Mental.

Introdução

O estresse é considerado experiência que traz tensão e/ou irritação em situações que provoque medo, excitação ou confusão, fazendo o organismo reagir aos componentes físicos ou psicológicos que o causaram. Atualmente, o termo estresse é empregado nos mais variados contextos ${ }^{(1)}$. Entretanto, profissões que exigem contato mais próximo com as pessoas, carregadas de envolvimento afetivo como medicina, psicologia, enfermagem e fisioterapia estão mais sujeitas ao desenvolvimento do estresse ${ }^{(2-4)}$.

A enfermagem é uma profissão considerada de risco para o desenvolvimento desse estado, uma vez que o aluno, , desde a formação acadêmica, se depara com situações que exigem tomadas de decisões importantes no cuidado do paciente. Ademais, a insegurança e a ansiedade, decorrentes desse processo, podem desencadear ou piorar os sintomas. Características como alto nível de habilidades cognitivas, disposição e atitudes proativas são constantemente requeridas tanto dos enfermeiros 
que atuam na prática como dos estudantes de enfermagem. Dessa forma, o estresse pode prejudicar o desempenho tanto no âmbito acadêmico, quanto na área assistencial. ${ }^{(3-5)}$.

Estudos que abordam o diagnóstico do estresse em estudantes de enfermagem, principalmente nos alunos do ensino superior ${ }^{(1)}$, mostram que a complexidade do curso e o lidar com os limites humanos podem frequentemente desencadear esse processo no estudante, por vezes, levando ao surgimento de condições crônicas em pessoas mais suscetíveis ${ }^{(1,6)}$. Logo, o fator "estresse" é um aspecto a ser levado em consideração, tendo em vista que estes serão os futuros profissionais e cuidadores, em parte responsáveis pelo tratamento, recuperação e reabilitação de uma clientela ${ }^{(7)}$.

Percebe-se que o estresse se tornou problema de saúde pública e é preciso mais atenção por parte da sociedade e das entidades promotoras de saúde para essa questão.Deste modo, , estudos devem ser realizados para identificação desse fator agravante e suas fontes, objetivando a qualidade de vida dos sujeitos $^{(7)}$.

Além disso, a escassez de pesquisas que abordam esse tema no universo dos estudantes de enfermagem é outro ponto a ser considerado, que comprova a importância da realização de novos estudos. A maioria dessas investigações utilizam uma metodologia qualitativa para analisar o estresse entre estudantes, e não instrumentos com propriedade psicométricas conhecidas. Isso pode ocorrer devido o estresse ser um fenômeno difícil de ser medido de maneira direta, sendo necessária a utilização de ferramentas de avaliação adequadas para uma correta identificação desse fenômeno ${ }^{(8)}$.

Portanto, o desenvolvimento de pesquisas que abordem esse tema é fundamental, pois o estresse não é somente um processo resultante da mudança dos hábitos e estilos de vida inadequados, mas, também, um fator de risco para outras doenças e distúrbios psíquicos, podendo ser causador de enfermidades no decorrer da vida. Além disso, influencia aqueles que convivem com os que sofrem de estresse, como familiares, amigos, colegas de trabalho, que estão envoltos no desenvolvimento dessa morbidade e, também, no apoio para sua resolução(7).

Com base no que foi exposto, surgiu o seguinte questionamento: Qual o nível de estresse entre acadêmicos de enfermagem? Desta forma, o presente estudo teve como objetivo estimar o nível de estresse e a sintomatologia apresentada entre acadêmicos de enfermagem de uma universidade pública federal.

\section{Método}

Trata-se de um estudo transversal e descritivo com abordagem quantitativa, envolvendo graduandos de enfermagem de uma universidade pública federal de João Pessoa/PB, realizada no período compreendido entre fevereiro e junho de 2014.

A amostra foi obtida a partir de uma lista de alunos regularmente matriculados do $3^{\circ}$ ao $10^{\circ}$ período do segundo semestre letivo de 2013, fornecida pela Secretaria do Curso de Enfermagem. Os alunos do $1^{\circ}$ e $2^{\circ}$ períodos foram excluídos devido à grade curricular ser composta por disciplinas que acontecem em diferentes prédios, tornando difícil sua localização. Considerando a população $(\mathrm{N}=314)$ e que o objetivo do presente estudo é estimar o nível de estresse entre os alunos, então o interesse recai em estabelecer uma proporção(9). Sendo assim, o cálculo da amostra foi estimado em 145 sujeitos, distribuídos nos oito períodos, conforme apresentado na tabela abaixo.

Tabela 1- Número de alunos selecionados por período. João Pessoa, PB, fevereiro a junho de 2014.

\begin{tabular}{ccc}
\hline Período & $\begin{array}{c}\text { Alunos } \\
\text { matriculados }\end{array}$ & Amostra \\
\hline $3^{\circ}$ & 46 & 21 \\
$4^{\circ}$ & 41 & 19 \\
$5^{\circ}$ & 43 & 20 \\
$6^{\circ}$ & 43 & 20 \\
$7^{\circ}$ & 37 & 17 \\
$8^{\circ}$ & 30 & 14 \\
$9^{\circ}$ & 38 & 18 \\
$10^{\circ}$ & 36 & 16 \\
\hline Total & 314 & 145 \\
\hline
\end{tabular}

A seleção dos alunos em cada período se deu a partir de um plano da amostra probabilístico aleatório simples, no qual foi realizado um sorteio a partir da listra de alunos matriculados em cada período. Apenas os alunos que não se encontravam no momento do sorteio foram excluídos, sendo realizado, na sequência, um novo sorteio para substituí-lo.

Considerando a possibilidade de perda em 5\% por rasura do questionário ou recusa em participar da pesquisa, foram selecionados aleatoriamente 152 alunos. Ao final, não foi registrado rasuras e apenas 
uma aluna se recusou a participar, totalizando uma amostra de $(n=151)$ graduandos de enfermagem.

Os alunos participantes da pesquisa foram abordados em sala de aula, antes do início ou depois das atividades acadêmicas, tendo em média 15 minutos para responder espontaneamente aos instrumentos e todos foram orientados a não rasurarem suas respostas.

Como instrumentos de coleta dos dados foram utilizados dois questionários. Um semiestruturado contendo dados socioeconômicos que aborda as seguintes variáveis: sexo, idade, cor, renda per capita, religião e ocupação ou profissão.

Para investigar o estresse entre os acadêmicos de enfermagem foi utilizado o Inventário de Sintomas do Stress para adultos de Lipp (ISSL)(10). O ISSL é um instrumento validado que identifica o estado de estresse, sua fase e vulnerabilidade à sintomatologia física, psicológica ou ambas. Composto por 52 itens, divididos em 3 quadros que avaliam os sintomas apresentados nas últimas 24 horas (Fase I - Alerta), no último mês (Fase II - Resistência) e nos últimos três meses (Fase III - Exaustão) (11).

$\mathrm{Na}$ Fase de alerta, o organismo tem uma excitação de agressão ou de fuga ao estressor, que pode ser entendida como um comportamento de adaptação. Nos dois casos, reconhece-se uma situação de reação saudável ao estresse, porquanto possibilita o retorno à situação de equilíbrio após a experiência estressante. A Fase de resistência é marcada pela persistência da fase de alerta, onde o organismo altera seus parâmetros de normalidade e concentra a reação interna em um determinado órgão alvo, desencadeando a síndrome de adaptação local (SAL). Na Fase de exaustão, o organismo encontrase extenuado pelo excesso de atividades, ocorrendo, então, a falência do órgão mobilizado na $S A L$, o que se manifesta sob a forma de doenças orgânicas ${ }^{(12)}$.

No entanto, outra fase do processo de estresse foi identificada, à qual foi dado o nome de Quase Exaustão, por se encontrar entre as fases de resistência e exaustão, provocando na pessoa uma sensação forte de esgotamento aumentando a chance de descontrole emocional. É importante lembrar que nem sempre a pessoa passa pelas quatro fases, e só alcançará a fase de exaustão quando o estressor for muito grave e não conseguir se adaptar à situação(12).

Os dados foram analisados descritivamente por meio de distribuições absolutas e percentuais. O programa utilizado para a digitação dos dados e obtenção dos cálculos estatísticos foi software estatístico $R$, na versão 2.12.1 $1^{(13)}$.

O presente estudo atendeu aos requisitos propostos pela Resolução $n^{\circ} 466 / 12$ de 12 de dezembro de 2012. Esse estudo é um desdobramento de um projeto maior, intitulado: Práticas de Cuidado no Sistema Formal e Informal de Saúde, aprovado pelo Comitê de Ética e Pesquisa da Universidade Federal da Paraíba, sob o número do protocolo 0059.

\section{Resultados}

A maioria dos alunos entrevistados era do sexo feminino $(90 \%)$ e mais da metade $(64,9 \%)$ tinham entre 20 e 25 anos de idade, sendo a média da idade de 22,74 anos. Declaram-se brancos $41,1 \%$ e não brancos $58,9 \%$. A religião católica teve o maior índice de ocorrência (64,2\%). Em relação à renda per capita, levou-se em consideração os valores calculados com base no salário mínimo vigente $(R \$ 724,00)$ em 2014 , e a partir disto constatou-se que $41,7 \%$ dos alunos declararam renda per capta de até um salário mínimo; $13,9 \%$ entre um e três salários e 44,4\% com renda maior que três salários. Quanto à ocupação ou profissão, dos 151 estudantes entrevistados, 10,6\% tinham a formação em técnico de enfermagem, porém desse percentual, apenas $8,6 \%$ exerciam a profissão, desempenhando as funções de aluno e profissional da saúde ao mesmo tempo.

Na Tabela 2 verifica-se que $49,7 \%$ dos alunos apresentam nível de estresse. No $9^{\circ}$ e $8^{\circ}$ períodos, a quantidade de indivíduos estressados aumentou em relação aos outros períodos, com respectivamente $7,9 \%$ e $7,2 \%$ dos alunos. Considerando-se que nos primeiros períodos a quantidade de pessoas sem estresse foi maior, nos $3^{\circ}, 4^{\circ}$ e $5^{\circ}$ períodos, com um percentil de $7,9 \%$ dos acadêmicos para cada um desses semestres letivos.

Tabela 2 - Distribuição dos alunos de enfermagem de acordo com período letivo cursado e presença de estresse segundo o ISSL. João Pessoa, PB, fevereiro a junho de 2014.

\begin{tabular}{ccccccc}
\hline Alunos de Enfermagem & \multicolumn{2}{c}{ Com Estresse } & \multicolumn{2}{c}{ Sem Estresse } & \multicolumn{3}{c}{ Total } \\
\hline Período Letivo & $\mathrm{N}$ & $\%$ & $\mathrm{~N}$ & $\%$ & $\mathrm{~N}$ & $\%$ \\
\hline $3^{\circ}$ & 09 & 6,0 & 12 & 7,9 & 21 & 13,9 \\
$4^{\circ}$ & 09 & 6,0 & 12 & 7,9 & 21 & 13,9 \\
& & & & & & (continua...)
\end{tabular}


Tabela 2 - continuação

\begin{tabular}{ccccccc}
\hline Alunos de Enfermagem & \multicolumn{2}{c}{ Com Estresse } & \multicolumn{2}{c}{ Sem Estresse } & \multicolumn{2}{c}{ Total } \\
\hline Período Letivo & $\mathrm{N}$ & $\%$ & $\mathrm{~N}$ & $\%$ & $\mathrm{~N}$ & $\%$ \\
\hline $5^{\circ}$ & 06 & 4,0 & 12 & 7,9 & 18 & 11,9 \\
$6^{\circ}$ & 09 & 6,0 & 11 & 7,3 & 20 & 13,3 \\
$7^{\circ}$ & 09 & 6,0 & 09 & 6,0 & 18 & 11,9 \\
$8^{\circ}$ & 11 & 7,2 & 06 & 4,0 & 17 & 11,2 \\
$9^{\circ}$ & 12 & 7,9 & 08 & 5,3 & 20 & 13,3 \\
$10^{\circ}$ & 10 & 6,6 & 06 & 4,0 & 16 & 10,6 \\
Total & 75 & 49,7 & 76 & 50,3 & 151 & 100,0 \\
\hline
\end{tabular}

De acordo com o ISSL, $49,7 \%$ dos alunos que estavam estressados, a maior parte desse percentual $(42,4 \%)$ se encontrava na fase de resistência, estando o maior número de acadêmicos distribuídos nos $8^{\circ} \mathrm{e}$ $9^{\circ}$ períodos (Tabela 3 ).

Tabela 3 - Distribuição dos alunos de enfermagem por fases do estresse, segundo o ISSL. João Pessoa, PB, fevereiro a junho de 2014.

\begin{tabular}{|c|c|c|}
\hline Fase & Frequência & Porcentagem \\
\hline Alerta & 04 & 2,7 \\
\hline Resistência & 64 & 42,4 \\
\hline Quase exaustão & 05 & 3,3 \\
\hline Exaustão & 02 & 1,3 \\
\hline Sem estresse & 76 & 50,3 \\
\hline Total & 151 & 100,0 \\
\hline
\end{tabular}

$\mathrm{Na}$ tabela 4 observa-se que os sintomas psicológicos são os mais presentes naqueles alunos que possuem estresse $(50,7 \%)$, seguido dos sintomas físicos $(40,0 \%)$. Na fase de Resistência ou Quase exaustão os sintomas psicológicos estavam presentes em $42,7 \%$ dos casos e os sintomas físicos em $40,0 \%$. Apenas $9,3 \%$ dos acadêmicos desenvolveram ambos os sintomas.

Tabela 4 - Distribuição dos alunos de enfermagem quanto ao tipo de sintoma de estresse por fase, segundo o ISSL. João Pessoa, PB, fevereiro a junho de 2014.

\begin{tabular}{lccccccccc}
\hline \multirow{2}{*}{ Fases } & \multicolumn{4}{c}{ Sintomas } & \multicolumn{2}{c}{ Total por fase } \\
\cline { 2 - 7 } & \multicolumn{2}{c}{ Físicos } & \multicolumn{1}{c}{ Psicológicos } & Físicos e Psicológicos & & \\
\hline & $\mathrm{N}$ & $\%$ & $\mathrm{~N}$ & $\%$ & $\mathrm{~N}$ & $\%$ & $\mathrm{~N}$ & $\%$ \\
\hline Alerta & 00 & 0,0 & 04 & 5,3 & 00 & 0,0 & 04 & 5,3 \\
Resistência ou quase exaustão & 30 & 40,0 & 32 & 42,7 & 07 & 9,3 & 69 & 92,0 \\
Exaustão & 00 & 0,0 & 02 & 2,7 & 00 & 0,0 & 02 & 2,7 \\
Total por desgaste & 30 & 40,0 & 38 & 50,7 & 07 & 9,3 & 75 & 100,0 \\
\hline
\end{tabular}

\section{Discussão}

O perfil dos graduandos de enfermagem encontrado neste estudo não difere dos encontrados em outras pesquisas. A maior parte deles é do sexo feminino. Essa diferença se deve ao fato de a profissão estar histórica e culturalmente ligada às práticas femininas. A enfermagem tem como objeto o cuidado, que sempre foi visto como uma qualidade hegemonicamente feminina. A mulher é preparada desde pequena para ser mãe, cuidar da casa, da família, dos enfermos, ou seja, cuidar de tudo e de todos. Apesar de tantas mudanças no mundo e nas profissões, a enfermagem continua sendo vista como uma profissão de característica feminina ${ }^{(7,14-15)}$. 
Referente à variável situação financeira, percebese que boa parte dos participantes do estudo possuem renda per capita baixa. Ter o tempo preenchido por inúmeras atividades acadêmicas, tendo em vista que o é curso integral, diminui as chances dos estudantes de conseguirem trabalhar, fator esse citado como estressor, da mesma maneira que a falta de dinheiro, acarretando dificuldades financeiras ${ }^{(7)}$.

Alguns dos acadêmicos investigados possuíam curso técnico de enfermagem. Um estudo realizado com o objetivo de analisar o perfil do estudante ingresso no curso de graduação em enfermagem, identificou que quase metade de sua amostra frequentou curso técnico antes de ingressar na universidade. Isso mostra a constante busca pela qualificação, item exigido em um mercado de trabalho altamente competitivo ${ }^{(15)}$.

No estudo em questão, quase metade dos discentes apresentaram nível de estresse. $O$ estresse é uma ocorrência usual e esperada no ambiente acadêmico de enfermagem. Os estudantes de cada instituição de ensino vivenciam em menor ou maior intensidade esse processo durante o período de ensino e aprendizagem e são dependentes da realidade em que vivem, pois, ocorrem flutuações nessa intensidade durante as distintas etapas da sua formação ${ }^{(16)}$. O estresse, quando instalado nesta população, pode gerar problemas favorecendo a diminuição do rendimento acadêmico e da qualidade de assistência prestada durante os estágios teóricopráticos $^{(17) .}$

Dentre os acadêmicos que apresentaram algum nível de estresse, a maioria se encontrava nos últimos períodos da graduação. Isto ocorre devido a natural preocupação com o desenvolvimento da carreira e da vida pessoal. Conteúdo programático das atividades acadêmicas teóricas e práticas, elaboração do trabalho de conclusão de curso, preocupações relacionadas à inserção no mercado de trabalho, relação trabalho/estudo/vida familiar, dificuldade no relacionamento interpessoal, avaliações, sobrecarga e atividades extracurriculares são considerados fatores estressores. Assim, é preciso aprender a conviver com o ritmo de vida agitado, desgastante, com falta de tempo para o lazer e descanso, alimentação inadequada e cobrança excessiva que são fatores fundamentais no desenvolvimento desse quadro de estresse $^{(7,17-18)}$.

No dia a dia do acadêmico de enfermagem, todos esses fatores citados são vivenciados, desde o momento do ingresso na universidade. Com curso em horário integral, o ritmo de vida intenso e a pressão psicológica causada pelas exigências impostas pelos professores e pela ansiedade em ter um bom rendimento a cada período cursado, aos poucos tornam-se fatores estressores aos alunos ${ }^{(7)}$.

Esse estudantes vivenciam no último ano de sua formação a transição da vida acadêmica para a laboral, que vem acompanhada do aumento de tarefas com alto grau de exigência e de responsabilidade, despertando tensões e ansiedade. Além disso, a carga horária de estudos e estágios coloca o aluno em condição de maior vulnerabilidade ${ }^{(19-21)}$.

A transição do período acadêmico para a nova fase de atuação profissional envolve importantes decisões, contribuindo para a presença de estresse, tais como: voltar para a cidade de origem ou enfrentar uma cidade grande; começar a trabalhar; constituir sua família eassumir responsabilidades financeiras. Assim, sair do ambiente acadêmico protegido e combater novos desafios pode ocasionar estresse e, por consequência, desequilíbrio emocional e estados de depressão(22).

A preocupação dos acadêmicos do último ano também pode envolver o sentimento de despreparo diante do mercado de trabalho, pois são praticamente profissionais e podem sentir medo de atuarem sozinhos, em breve, sem o respaldo do professor. Muitas vezes, esse processo é acompanhado de competitividade entre os próprios colegas ${ }^{(19-20)}$.

A identificação de fatores de estresse deve proporcionar aos estudantes um processo de reflexão de suas atividades, da mesma maneira que contribuir para a elaboração de estratégias de gerenciamento das situações desgastantes que possam surgir no último ano do curso, levando a um melhor aproveitamento acadêmico e preparando-os para enfrentar um mercado de trabalho com importantes desafios como futuros profissionais ${ }^{(17)}$.

O atual estudo revelou que a maioria dos discentes encontrava-se na fase de resistência de acordo com o ISSL. Hans Selye foi o primeiro cientista a utilizar o termo estresse na área da saúde,descrevendo, através de suas observações, a síndrome geral de adaptação (SAG), que pode ser entendida como o conjunto das reações do organismo frente à exposição prolongada do estressor. Segundo este autor, essa síndrome apresenta três fases ou estágios ${ }^{(23)}$.

Nesta pesquisa verificou-se que mais da metade dos participantes apresentaram sintomas psicológicos decorrentes do estresse. As situações potencialmente estressoras se refletem na qualidade de vida desses alunos, que podem vir a paresentar sintomas decorrentes desse processo ${ }^{(24)}$. 
Outro estudo qualitativo sobre o estresse entre acadêmicos de enfermagem demonstrou significado pessoal para os estudantes, trazendo como categoria estudada o estresse como manifestação física e mental. Os participantes relataram a presença de corpo e mente cansados, irritabilidadefrequente, pessimismo, mau humor, antissocialismo e

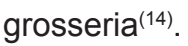

Os estudantes de enfermagem apresentam manifestações psicológicas e fisiológicas de estresse em maior proporção que os de outras áreas. Portanto, é importante investigar as manifestações e fontes de estresse, para que possam ser traçadas estratégias de intervenção, com o objetivo de proporcionar melhoria na qualidade de vida dessesindivíduos. Os alunos treinados para utilizar estratégias de combate usufruirão melhor a etapa acadêmica e aplicarão a experiência na futura vida profissional| ${ }^{(1,24-25)}$.

Ressalta-se a necessidade de atenção aos educadores e a criação de programas com ações preventivas e terapêuticas que possibilitem a reflexão e o encontro de saídas saudáveis para suas angústias, aprendendo a utilizar o conhecimento adquirido no seu desempenho futuro(22).

É necessário que as Instituições de Ensino Superior tenham meios de apoiar os estudantes em uma vida acadêmica mais saudável, uma vez que os graduandos passam pormomentos de transformações, crescimentos, fracassos, amadurecimentos, medo, ansiedade vivenciados em espaços clínicos ou de salas de aulas.. Assim, o local que deveria favorecer a construção do conhecimento e ser o alicerce para as suas experiências de formação profissional, pode tornar-se desencadeador de patologias ${ }^{(6)}$.

\section{Conclusão}

Espera-se que os resultados encontrados neste estudo possam ampliar as possibilidades de melhoria na organização dos cursos de graduação em enfermagem, com o objetivo de tornar o ambiente acadêmico menos desgastante. É importante a implementação de estratégias que amparem o estudante, uma vez que a presença do estresse pode comprometer o seu aprendizado e desempenho, influenciando diretamente na assistência prestada por esses indivíduos e na sua qualidade de vida..

Faz-se necessário ofertar aos alunos melhores condições de combate ao estresse, sendo imperiosa a participação direta dos docentes e da instituição na elaboração ou implementação de estratégias para que esse objetivo seja alcançado. Recomenda-se, a partir do presente estudo, a implementação de ferramentas de tecnologia leve do cuidado, que apresentam baixo custo e alto poder resolutivo, como disponibilização de informações sobre o estresse, sua prevenção e controle, sessões de relaxamento, terapia comunitária integrativa, espaços para atividade física orientada por profissionais capacitados, por exemplo.

Além da implementação dessas tecnologias, sugere-se que novos estudos sejam realizados com o objetivo de descobrir novas ferramentas para o planejamento, desenvolvimento e busca de aprimoramento do processo ensino e aprendizagem, assim como a investigação de melhores formas de prevenção do estresse para os acadêmicos de enfermagem.

\section{Referencias}

1. Phun ET, Santos CB. Consumo de álcool e estresse em estudantes do segundo ano de enfermagem. Rev. Latino-Am. Enfermagem. [Internet]. 2010 [Acesso 3 mai 2015];18(spe):496-503. Disponível em: http:// www.scielo.br/scielo.php?script=sci_arttext\&pid =S0104-11692010000700003.

2. Ribeiro RP, Martins JT, Marziale MHP, Robazzi MLCC. Work-related illness in nursing: an integrative review. Rev Esc Enferm USP. [Internet]. 2012 [Accessed 2015 Mai 03];46(2):495-504. Available from: $\quad$ http://www.scielo.br/scielo.php?pid=S008062342012000200031\&script=sci_arttext\&tlng=en.

3. Fanco Jr GP, Barros ALBL, Nogueira-Martins LA, Zeitoun SS. Burnout in nursing residents. Rev Esc Enferm USP. [Internet]. 2012 [Accessed 2015 Mai 07];45(1):12-8. Available from: http:// www.scielo.br/readcube/epdf.php?doi $=10.1590 /$ S $0080-62342011000100002 \&$ pid = S 0080 62342011000100002\&pdf_path=reeusp/v45n1/en_02. pdf\&lang=en.

4. Rodrigues VM, Ferreira ASS. Stressors in nurses working in Intensive Care Units. Rev. Latino-Am. Enfermagem. [Internet]. 2011 [Accessed 2015 Mai 09];19(4):1025-32. Available from: http:// www.scielo.br/readcube/epdf.php?doi $=10.1590 /$ S $0104-11692008000100005 \& p i d=S 0104$ $11692008000100005 \&$ pdf_path $=$ rlae/v16n1/04. pdf\&lang=en.

5. Lorenz VR, Benatti MCC, Sabino MO. Burnout and stress among nurses in a university tertiary hospital. Rev. Latino-Am. Enfermagem. [Internet]. 2010 [Accessed 2015 mai 07];18(6):1084-91. Available from: http:// www.scielo.br/readcube/epdf.php?doi $=10.1590 /$ S0104-11692010000600007\&pid=S0104 - 
$11692010000600007 \& p d f \_p a t h=r l a e / v 18 n 6 / 07$. pdf\&lang=en.

6. Oliveira BLCA, Ferreira MO Filha, Monteiro $\mathrm{CH}$, Monteiro RVP, Cunha CLF. Estresse entre graduandos de enfermagem de uma universidade pública federal: um estudo epidemiológico. J Manag Prim Health Care. [Internet]. 2012 [Acesso 3 maio 2015];3(2):72-9. Disponível em: http://jmphc.com/ojs/index.php/01/article/ view/43/42.

7. Pereira CA, Miranda LCS, Passos JP. O estresse e seus fatores determinantes na concepção dos graduandos de enfermagem. REME Rev Min Enferm. [Internet]. 2010 [Acesso 2015 mai 11];14(2): 204-9. Disponível em: http:// www.reme.org.br/exportar-pdf/107/v14n2a09.pdf.

8. Costa ALS, Polak C. Construccion and validation of and instrument for the assessment of stress among nurse students. Rev Esc Enferm USP. [Internet]. 2009 [Accessed 2015 jun 15];43(Esp):1017-26. Available from: http://www.scielo.br/readcube/epdf.php?doi=10.1590/ s $0080-62342009000500005 \& \mathrm{pid}=\mathrm{s} 0080-$ $62342009000500005 \&$ df_path=reeusp/v43nspe/en_ a05v43ns.pdf\&lang=en.

9. Campbell D, Stanley J. Delineamentos Experimentais e Quase-experimentais da Pesquisa. São Paulo: EDUSP; 1979.

10. Lipp MEN, Guevara AJH. Validação empírica do Inventário de Sintomas de Stress. Estud Psico. 1994;11(3):43-9.

11. Minari MRT, Souza JC. Stress em servidores públicos do instituto nacional de seguro social. Estud Psicol. [Internet]. 2011 [Acesso 7 jun 2015];28(4):5218. Disponível em: http://www.scielo.br/scielo. php?script=sci_arttext\&pid=S0103-166X201100040001 $2 \& \operatorname{lng}=$ en\&tlng=pt.

12. Lipp MEN. Stress: conceitos básicos. In: Lipp MEN, editor. Pesquisas sobre stress no Brasil: saúde, ocupações e grupos de risco. Campinas (SP): Papirus; 1996.

13. R Development Core Team. R: A language and environment for statistical computing, reference index version 2.12.1. R Foundation for Statistical Computing. [Internet]. 2010 [Accessed 2014 Feb 15]. Vienna, Austria. Available from: http://www.R-project.org.

14. Corral-Mulato S, Baldissera VDA, Santos JL, Philbert LAS, Bueno SMV. Estresse na vida do acadêmico em enfermagem: (Des)conhecimento e prevenção. Invest Educ Enferm. [Internet] 2011 [Acesso 15 jun 2015];29(1):109-17. Disponível em: http://www.scielo. org.co/pdf/iee/v29n1/v29n1a14.pdf.

15. Donati L, Alves MJ, Camelo SHH. O perfil do estudante ingressante no curso de graduação em enfermagem de uma faculdade privada. Rev Enferm UERJ. [Internet] 2010 [Acesso 21 jun 2015];18:446-50. Disponível em: http://www.facenf.uerj.br/v18n3/v18n3a19.pdf.
16. Edwards D, Burnard P, Bennett K, Hebden U. A longitudinal study of stress and self-esteem in student nurses. Nurse Educ Today. 2010;30(1):78-84.

17. Silva VLS, Chiquito NC, Andrade RAPO, Silva MFP, Camelo $\mathrm{SHH}$. Fatores de estresse no último ano do curso de graduação em enfermagem: percepção dos estudantes. Rev Enferm UERJ. [Internet] 2011 [Acesso 4 jun 2015];19(1):121-6. Disponível em: http://www.facenf. uerj.br/v19n1/v19n1a20.pdf.

18. Amaducci CM, Mota DDFC, Pimenta CAM. Fatigue among nursing undergraduate students. Rev Esc Enferm USP. [Internet] 2010 [Accessed 2015 Mai 25];44(4):1052-8. Available from: http:// www.scielo.br/readcube/epdf.php?doi=10.1590/ S $0080-62342010000400028$ \& pid=S 0080 $62342010000400028 \&$ pdf_path=reeusp/v44n4/en_28. pdf\&lang=en.

19. Furegato ARF, Santos JLF, Silva EC. Depressão entre estudantes de dois cursos de enfermagem: autoavaliação da saúde e fatores associados. Rev Bras Enferm. [Internet] 2010 [Acesso 13 maio 2015];63(4):509-16. Disponível em: http://www.scielo.br/pdf/reben/v63n4/02.pdf.

20. Santos VEP, Radünz V. O estresse de acadêmicas de enfermagem e a segurança do paciente. Rev Enferm UERJ. [Internet] 2011 [Acesso 10 maio 2015];19(4):61620. Disponível em: http://www.facenf.uerj.br/v19n4/ v19n4a19.pdf.

21. Soares $\mathrm{MH}$, Oliveira FS. The relation between alcohol, tobacco and stress in nursing students. SMAD, Rev Eletrônica Saúde Mental Álcool Drog. [Internet] 2013 [Accessed 2015 Jun 22];9(2):88-94. Available from: http:// www.revistas.usp.br/smad/article/view/79661/83667

22. Moreira DP, Furegato ARF. Stress and depression among students of the last semester in two nursing courses. Rev. Latino-Am. Enfermagem. [Internet] 2013 [Accessed 2015 Jun 05];21(Spec):155-62. Available from: http://www.scielo.br/pdf/rlae/v21nspe/20.pdf

23. Selye H. The stress of life. New York: Mc Graw Hill; 1956.

24. Bublitz S, Guido LA, Freitas EO, Lopes LFD. Estresse em estudantes de enfermagem: uma revisão integrativa. Rev Enferm UFSM. [Internet] 2012 [Acesso 13 jun 2015];2(3):530-8. Disponível em: file:///C:/Users/Ricardo/ Downloads/3485-33508-1-PB.pdf.

25. Benavente SBT, Costa ALS. Physiological and emotional responses to stress in nursing students: an integrative review of scientific literature. Acta Paul Enferm. [Internet] 2011 [Accessed 2015 Jun 19];24(4):571-6. Available from: http://www.scielo.br/pdf/ape/v24n4/en_ a19v24n4.pdf

Recebido: 06.07.2015 Aprovado: 29.02.2016 